\title{
Research Paper \\ Comparison of Coping Styles, Distress Tolerance and Religious Adherence in Mothers of Children with Physical-Motor Disabled and Normal
}

\author{
Zahra Rabani ${ }^{1, *}$ \\ ${ }^{1}$ MA of Social Research, Department of Social Studies, Dehaghan Branch, Islamic Azad University, Dehaghan, Iran \\ * Corresponding author: Zahra Rabani, Department of Social Studies, Dehaghan Branch, Islamic Azad University, Dehaghan, \\ Iran. E-mail: hastihasti6040@gmail.com \\ How to Cite this Article: \\ Rabani Z. Comparison of Coping Styles, Distress Tolerance and Religious Adherence in Mothers of Children with Physical-Motor \\ Disabled and Normal. J North Khorasan Univ Med Sci. 2020;6(4):10-18. \\ DOI: 10.29252 /ijrn-06042
}

\begin{tabular}{l}
\hline Received: 26 Jun 2019 \\
Accepted: 15 Jan 2020 \\
\hline Keywords: \\
Coping Styles \\
Disabled \\
Distress Tolerance \\
Physical-Motor \\
Religious \\
\hline ○ 2020 Iranian Journal of \\
Rehabilitation Research in Nursing
\end{tabular}

\begin{abstract}
Introduction: Having a disabled child can negatively effect on health and quality of life of parents, especially mothers. Therefore, the present research aimed at the comparison of coping styles, distress tolerance, and religious adherence in mothers of children with physical-motor disabled and normal.

Methods: In a causal-comparative study among mothers of children with physicalmotor disabled and normal of Tehran city in the 2018 year, several 70 people (35 in each group) were selected by the available sampling method. The groups responded to the questionnaires of coping styles, distress tolerance, and religious adherence. Data were analyzed by multivariate analysis of variance method using SPSS version 19 software.

Results: The findings showed that there was a significant difference between the groups in terms of coping styles and distress tolerance, but there wasn't a significant difference in terms of religious adherence. In other words, the rate of distress tolerance and coping techniques, including problem-solving, physical control, attracting social support, emotional monitoring and cognitive evaluation in mothers with physical-motor disabled children were lower than mothers with healthy children $(\mathrm{P}<0.05)$.

Conclusions: The findings indicated there were lower types of coping styles, and distress tolerance in mothers of physical-motor disabled children is compared with mothers of healthy children. Therefore, specialists and authorities should design and implement programs to improve the coping styles and distress tolerance of mothers with physical-motor disabled children.
\end{abstract}

\section{Extended Abstract}

\section{OBJECTIVE}

$\mathrm{D}$ isability is the permanent inability to perform all or part of the ordinary necessities of individual or social life (1). Having a disabled child can negatively effect on health and quality of life of parents, especially mothers (3). Usually, mothers with disabled children are in a lower position in terms of coping styles than mothers with typical children (4). Also, distress tolerance means the capacity to resist negative emotions or tolerate negative inner states caused by stressors (8), and religious adherence can change one's attitude toward the world and increase one's ability to adapt to adverse, stressful and unpredictable circumstances (14). Therefore, the present research aimed at the comparison of coping styles, distress tolerance, and religious adherence in mothers of children with physical-motor disabled and normal.

\section{MATERIALS AND METHODS}

This research was a causal-comparative study. The statistical population was mothers of children with physical-motor disabled and normal of Tehran city in the 2018 year. The sample research was 70 people (35

Copyright (C) 2020 The Author(s); Published by Iranian Journal of Rehabilitation Research in Nursing. This is an open access article, distributed under the terms of the Creative Commons Attribution-NonCommercial 4.0 International License (http://creativecommons.org/licenses /by-nc/4.0/) which permits others to copy and redistribute material just in noncommercial usages, provided the original work is properly cited. 
in each group) who were selected by the available sampling method. The groups responded to the questionnaires of Billings and Moss's coping styles, Simons and Gaher's distress tolerance, and Janbozorgi's religious adherence. Data were analyzed by multivariate analysis of variance method using SPSS version 19 software.

\section{RESULTS}

The findings showed that there was no significant difference between the experimental and control groups in terms of education and age. Other results showed that there was a significant difference between the groups in terms of coping styles and distress tolerance. Still, there wasn't a significant difference in terms of religious adherence. In other words, the rate of distress tolerance and coping styles, including problem-solving, physical control, attracting social support, emotional monitoring and cognitive evaluation in mothers with physicalmotor disabled children was lower than mothers with typical children $(\mathrm{P}<0.05)$ (Tables 1-2).

Table 1. Mean and Standard Deviation of Variables

\begin{tabular}{lcc}
\hline Variables & Mothers with Disabled Children & Mothers with Normal Children \\
\hline Problem solving style & $4.35 \pm 0.74$ & $5.88 \pm 0.89$ \\
Physical control style & $12.41 \pm 2.08$ & $16.52 \pm 2.44$ \\
Attracting social support style & $6.28 \pm 1.14$ & $8.16 \pm 1.37$ \\
Emotional control style & $14.30 \pm 2.27$ & $19.31 \pm 2.92$ \\
Cognitive evaluation style & $9.22 \pm 1.88$ & $11.75 \pm 2.16$ \\
Distress tolerance & $37.26 \pm 3.39$ & $44.80 \pm 4.05$ \\
Distress tolerance & $121.43 \pm 11.75$ & $115.94 \pm 11.93$ \\
\hline
\end{tabular}

Data are presented as mean \pm SD.

Table 2. MANOVA Results

\begin{tabular}{lcccccc}
\hline Variables & Sum of Squares & Df & Mean Squares & F & P-Value & Eta Squared \\
\hline Poblem solving style & 33.739 & 1 & 33.739 & 16.169 & $<0.001$ \\
Physical control style & 115.864 & 1 & 115.864 & 29.565 & $<0.001$ \\
Attracting social support style & 43.864 & 1 & 43.864 & 22.750 & $<0.001$ \\
Emotional control style & 137.233 & 1 & 137.233 & 27.329 & $<0.001$ & 0.371 \\
Cognitive evaluation style & 62.280 & 1 & 62.280 & 19.638 & 0.483 \\
Distress tolerance & 394.209 & 1 & 394.209 & 12.750 & $<0.001$ \\
Distress tolerance & 1283.365 & 1 & 1283.365 & 1.461 & 0.347 & $<0.001$ \\
\hline
\end{tabular}

\section{CONCLUSION}

Due to the high number of children with disabilities and the problems that have their mothers, intervention is necessary to improve their characteristics. The findings indicated there were lower types of coping styles, and distress tolerance in mothers of physical-motor disabled children is compared with mothers of healthy children. Therefore, specialists and authorities should design and implement programs to improve the coping styles and distress tolerance of mothers with physical-motor disabled children.

\section{Ethical Considerations}

In this study, moral points according to the ethical protocols of Helsinki studies were done and received ethical code from the Islamic Azad University of Dehaghan branch with the number of IR.IAUDehaghan.REC.1397.41. Also, the research consent form was signed by all subjects.

\section{Funding or Supports}

This research has not sponsored and is done at personal expense.

\section{Author's Contribution}

Zahra Rabani collected data, analyzed them, and wrote the article.

\section{Conflict of Interest}

In this article, there was no reported any conflict of interest.

\section{Applicable Remarks}

Disables make the most significant minority of Iran and knowing them. Their parents, especially mothers who are considered primary and directly responsible for their care, have to need a lot of research. The results of the present study showed that distress tolerance and coping styles of mothers with disabled children were at the unsuitable level than those of mothers with healthy children, and there was no difference between them in terms of religious adherence. Therefore, health professionals can make based on results of this study and similar studies a perspective for improving the health of mothers with disabled children and design programs to enhance psychological characteristics especially to improving their distress tolerance and coping styles which use of such programs can be useful in improving the health-related attributes of mothers with disabled children.

\section{Acknowledgments}

Thus gratitude and appreciation from officials of the Islamic Azad University of Dehaghan branch and Welfare organization of Tehran city due to cooperation in conducting the research and from participants for participating in the study. 


\section{مقايسه سبكهاى مقابلهاى، تحمل يريشانى و يايبندى مذهبى در مادران داراى كودى معلول جسمى - حر كتى و عادى}

* نويسنده مسئول: زهرا ربانى، گَروه مطالعات اجتماعى، واحد دهاقان، دانشگاه آزاد اسلامى، دهاقان، ايران. ايميل: hastihasti6040@gmail.com

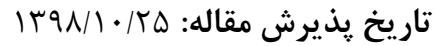

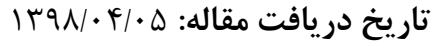

جكيده

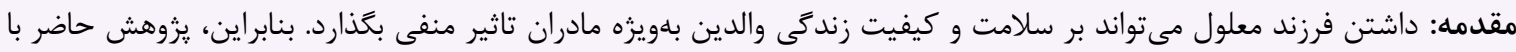

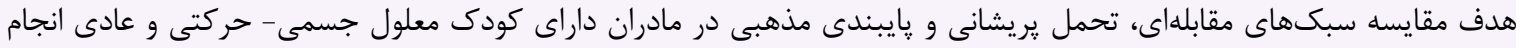

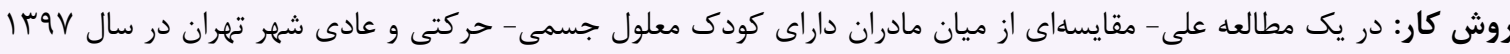

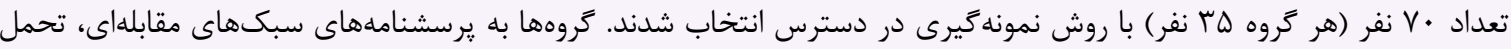

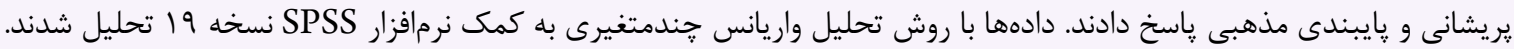

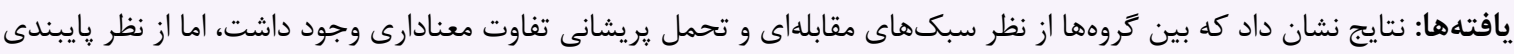

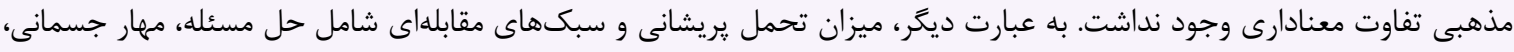

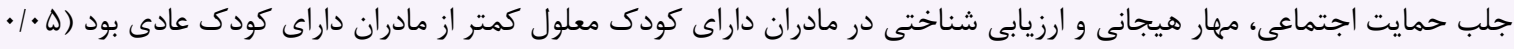
. $(\mathrm{P}<$

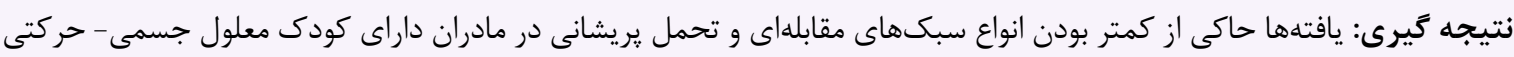

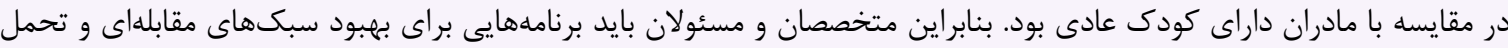

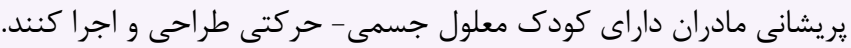

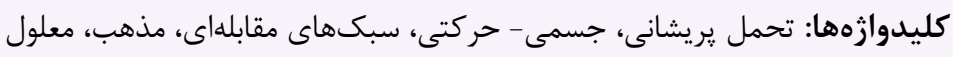

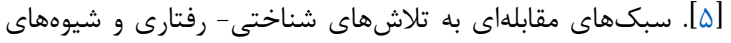

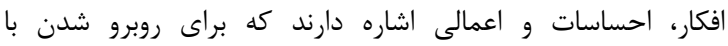

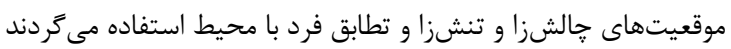

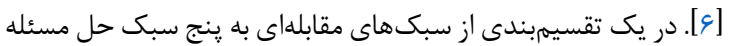

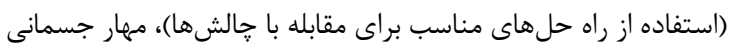

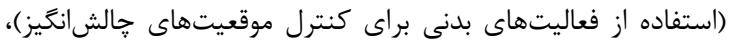

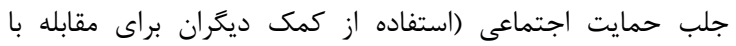

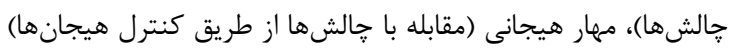

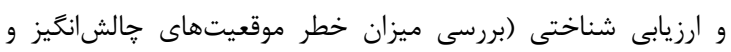

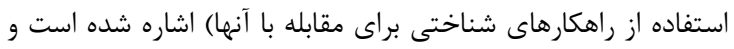

معلوليت ناتوانى دائمى در انجام تمام يا بخشى از ضروريات عادى زندكى إنى

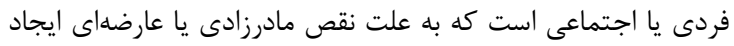

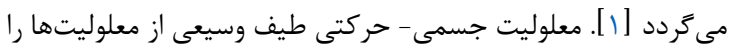

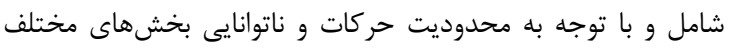

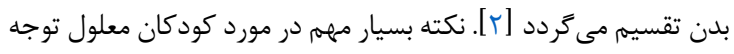

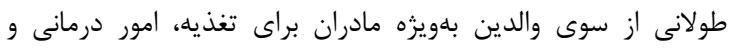

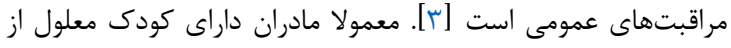

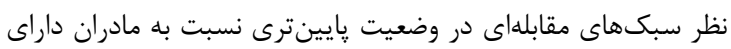

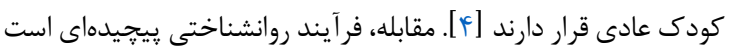
كه براى رويارويى با تنش، تصميمَّيرى و حل مسئله استفاده مى شود 
بيشتر از مادران دختران شنوا و ميزان تنظيم هيجان آنها كمتر بود

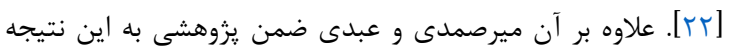

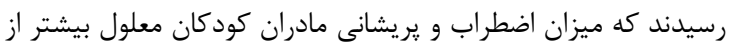

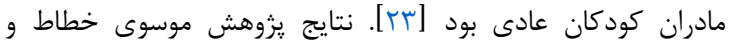

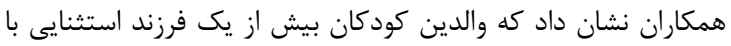

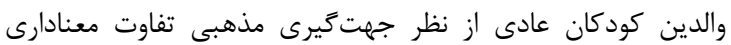

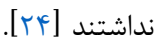

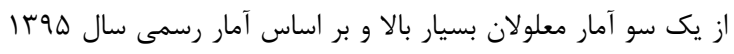

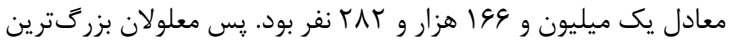

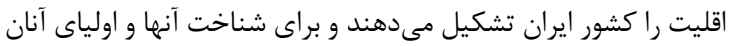

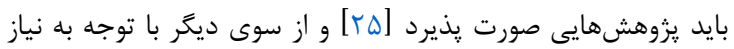

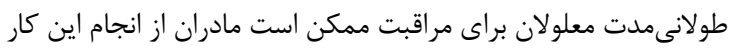

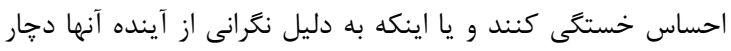

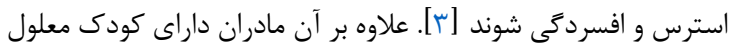

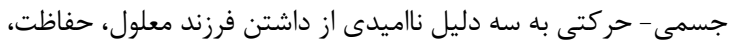

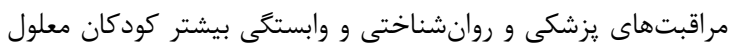

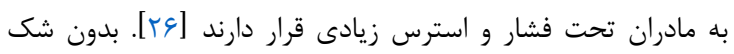

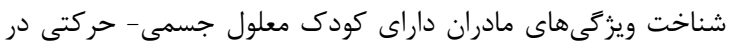

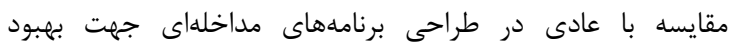

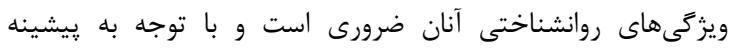

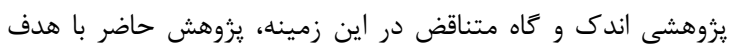

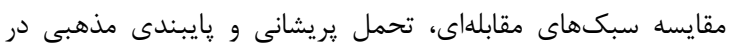
مادران داراى كودى معلول جسمى - حركتى و عادى انجام شد.

\section{روش كار}

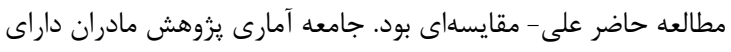

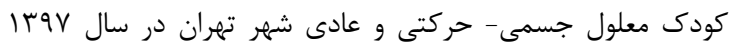

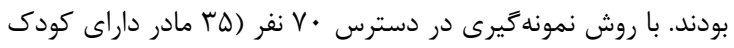

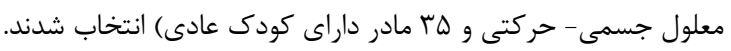

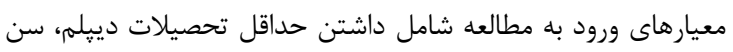

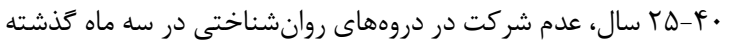

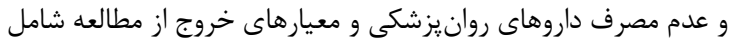

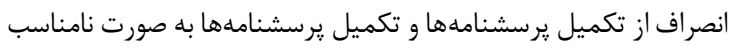

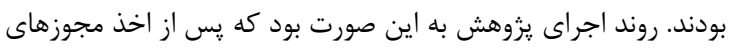

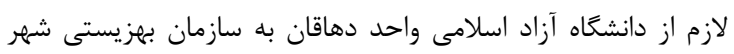

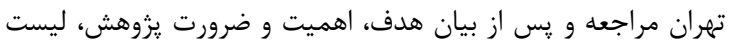

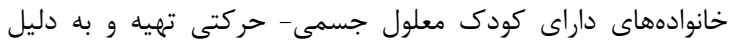

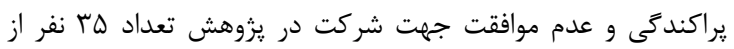

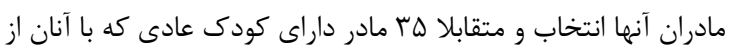

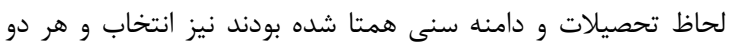

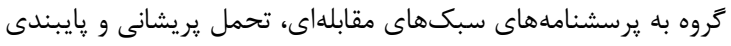
مذهبى ياسخ دادند.

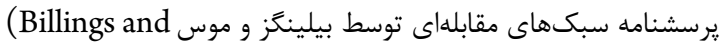

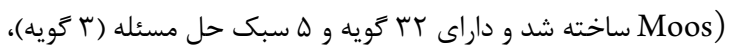

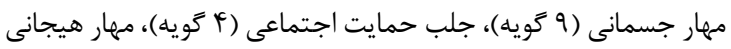

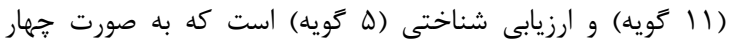

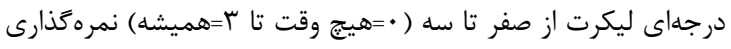

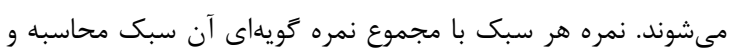

استفاده مناسب و كارآمد از آنها باعث افزايش سلامت، بهزيستى و

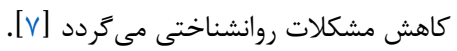

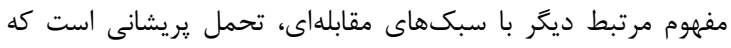

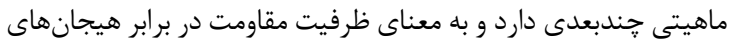

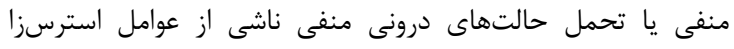

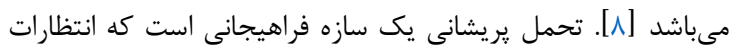

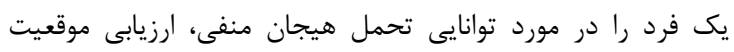

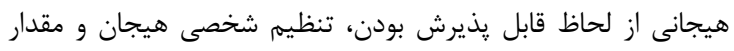

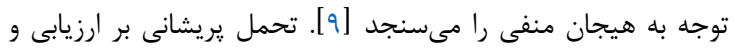

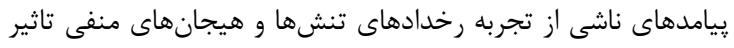

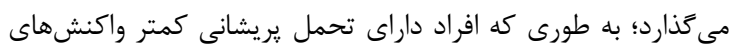

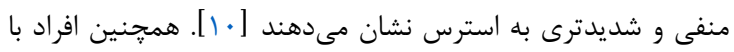

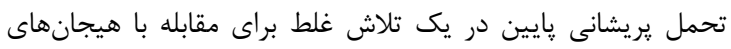

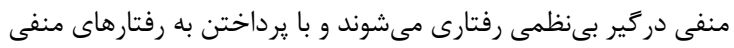

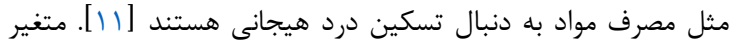

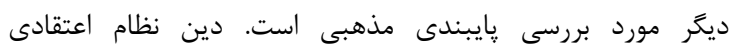

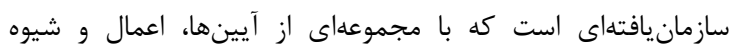

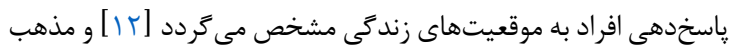

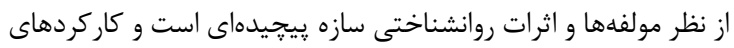

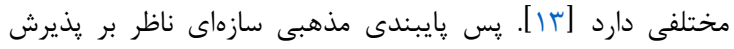

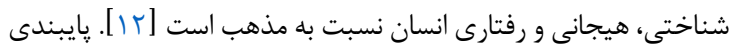

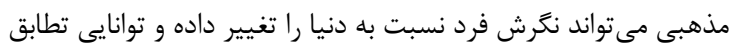

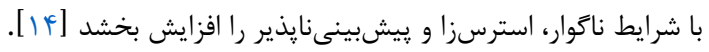

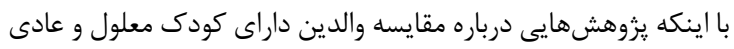

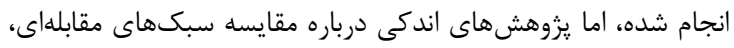

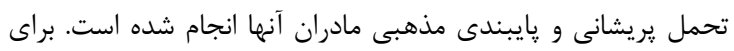

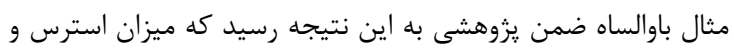

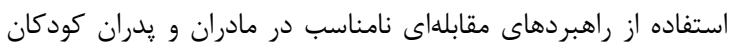

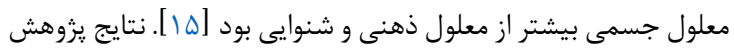

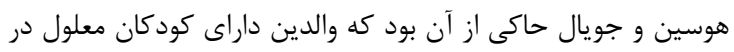

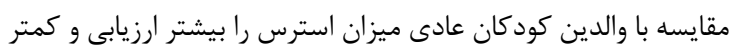

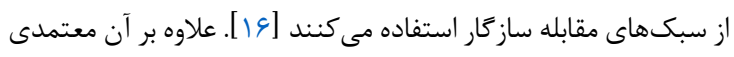

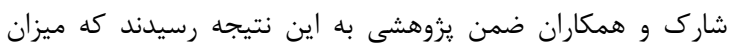

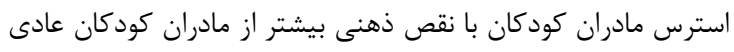

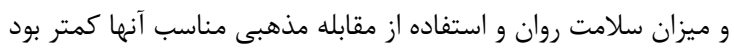

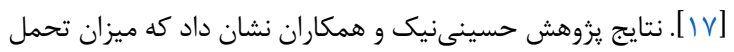

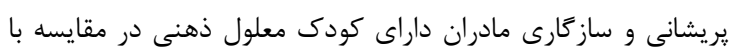

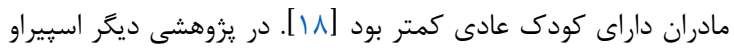

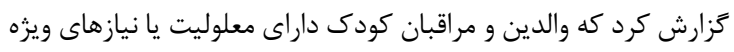

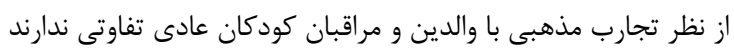

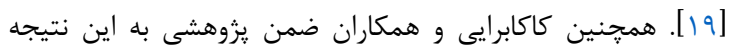

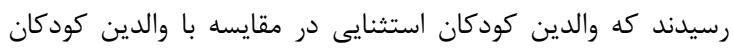

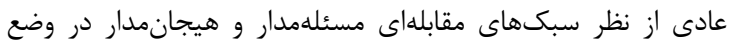

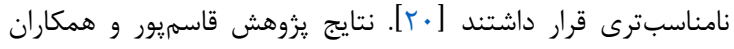

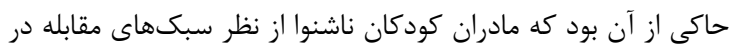

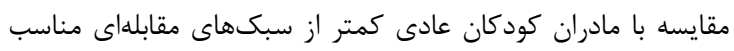

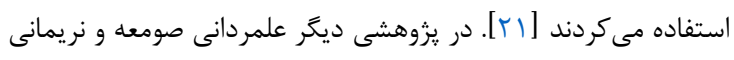
كزارش كردند كه ميزان عدم تحمل بلاتكليفى مادران دختران ناشني 
مخالف تا F=كاملا موافق) نمره كذارى مىشود. نمره ابزار با مجموع نمره

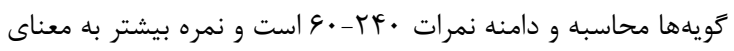

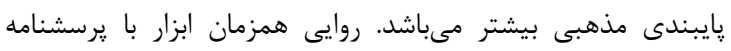

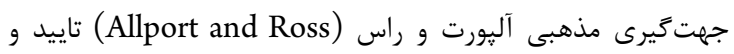

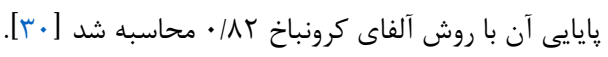
در مطالعه حاضر رعايت نكات اخلاقى توسط دانشعاه آزاد اسلامى واحد الند

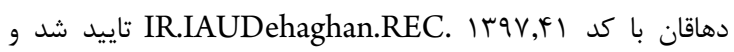

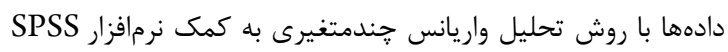
نسخه 19 تحليل شدند.

\section{|افتهها}

فراوانى مطلق و درصد فراوانى ويزگگىهاى جمعيتشناختى تحصيلات

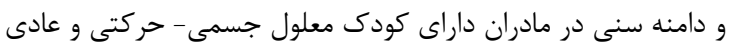

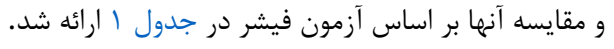

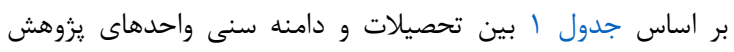

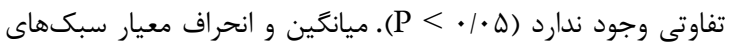

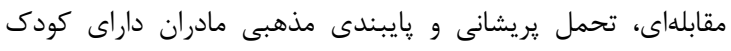

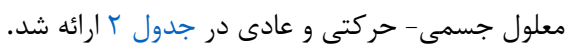

دامنه نمرات حل مسئله 9-•، مهار جسمانى YY-Y-•، جلب حمايت

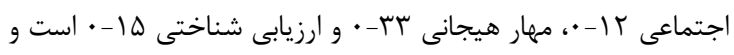

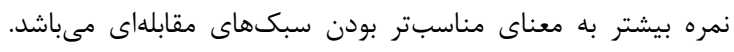

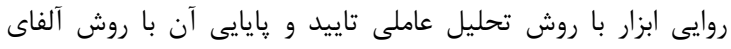

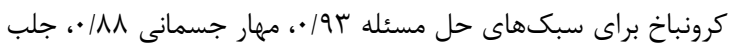

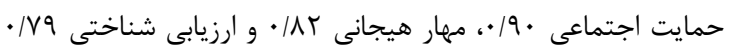

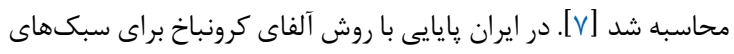

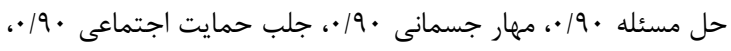

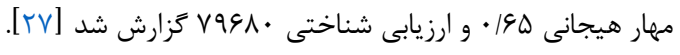

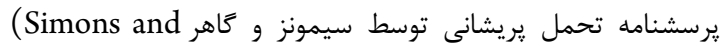

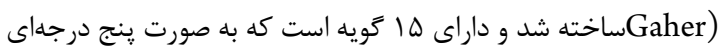

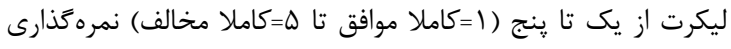

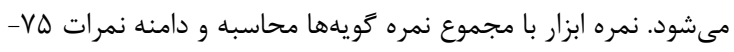

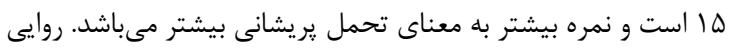

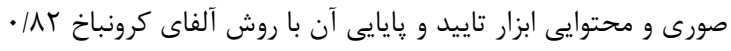

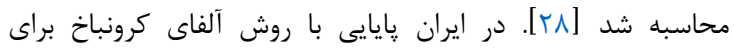

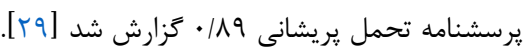

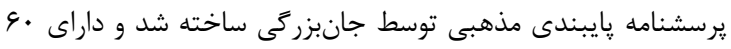
كويه است كه به صورت جهار درجهاى ليكرت از يك تا جهال (

\begin{tabular}{|c|c|c|c|c|c|}
\hline \multirow[t]{2}{*}{ P-Value Fisher } & \multicolumn{2}{|c|}{ مادران داراى كودى عادى } & \multicolumn{2}{|c|}{ مادران داراى كودى معلول } & \multirow[t]{2}{*}{ متغيرها } \\
\hline & درصد فراوانى & فراوانى & درصد فراوانى & فراوانى & \\
\hline \multirow[t]{5}{*}{$>\cdot 1 \cdot 0$} & & & & & تحصيلات \\
\hline & $r \Delta / V I$ & 9 & $r N / \Delta V$ & 1. & ميبيلم \\
\hline & Tr/NG & $\wedge$ & IVIIF & 4 & كاردانى \\
\hline & FT/AS & 10 & $\Psi N / \Delta V$ & iv & كارشناسى \\
\hline & $N / \Delta V$ & r & $\Delta / V I$ & r & كارشناسى ارشد \\
\hline \multirow[t]{4}{*}{$>\cdot 1 \cdot \Delta$} & & & & & دامنه سنى (سال) \\
\hline & TN/QV & 1. & TF/TA & ir & $r \cdot-r q$ \\
\hline & $r \Delta / V 1$ & 19 & FT/AS & 10 & $r \Delta-r 1$ \\
\hline & $r \Delta / v 1$ & 9 & Tr/AS & $\wedge$ & l. $-r_{q}$ \\
\hline
\end{tabular}

\begin{tabular}{|c|c|c|c|c|}
\hline \multicolumn{2}{|c|}{ مادران داراى كودى عادى } & \multicolumn{2}{|c|}{ مادران داراى كودى معلول جسمى - حركتى } & \multirow[t]{2}{*}{ متغيرها/تروهها } \\
\hline انحراف معيار & ميانغين & انحراف معيار & ميانكين & \\
\hline$\cdot / 19$ & $\Delta / \wedge \Lambda$ & $\cdot / V^{F}$ & $r / T \Delta$ & سبك حل مسئله \\
\hline r/AF & $\mid g / \Delta T$ & $r / \cdot \Lambda$ & $|r / 4|$ & سبك مهار جسمانى \\
\hline $1 / \pi v$ & $1 / 19$ & $1 / 14$ & $9 / \pi \Lambda$ & سبك جلب حمايت اجتماعى \\
\hline r/9 & $19 / \pi 1$ & $r / T V$ & $14 / \pi$ & سبك مهار هيجانى \\
\hline$r / 19$ & $11 / v \Delta$ & $1 / 1 \wedge$ & $9 / \pi r$ & سبك ارزيابى شناختى \\
\hline$F / \cdot \Delta$ & $r q / \lambda$. & $r / r q$ & rV/Te & تحمل بريشانى \\
\hline $11 / 94$ & $11 Q / 9 F$ & $11 / v \Delta$ & $|r| / F T$ & هايبندى مذهبى \\
\hline
\end{tabular}

\begin{tabular}{|c|c|c|c|c|}
\hline 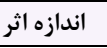 & P-Value & FريبF & مقدار & آزمونها \\
\hline - MFG & $\cdot 1 . \cdot 1$ & IT/NFT & $\cdot / F T \Lambda$ & اثر پِيلايى \\
\hline$\cdot / r \Delta \Lambda$ & $\cdot 1 . .1$ & IT/ATE & $\cdot / 9 T \Delta$ & لامبداى ويلكز \\
\hline$\cdot / T V \Delta$ &.. .1 & $|r / V \Delta|$ & . /4q4 & اثر هاتلينَ \\
\hline$\cdot \mid \pi Y \Lambda$ & $.1 . .1$ & $|N| \& \& V$ & $\cdot|4|$ & بزركترين ريشه روى \\
\hline
\end{tabular}




\begin{tabular}{|c|c|c|c|c|c|c|}
\hline اندازه اثر & P-Value & ضريبF & ميانغين مجذورات & درجه آزادى & مجموع مجذورات & متغيرها \\
\hline$\cdot / T M A$ & $.1 \cdot 1$ & $19 / 199$ & ( & 1 & Tr/vrq & سبك حل مسئله \\
\hline$\cdot 10 \cdot 9$ & $.1 . .1$ & $r q / \Delta \& \Delta$ & $\| \Delta / \Lambda G 4$ & 1 & $\| \Delta / \Lambda G 4$ & سبكى مهار جسمانى \\
\hline$\cdot|r v|$ & $.1 \cdot 1$ & TT/VQ. & $\langle r / 911$ & 1 & $F r / 911$ & سبك جلب حمايت اجتماعى \\
\hline . /4Ar & $\cdot . .1$ & TV/TTQ & ITV/Trt & 1 & ITV/Trt & سبك مهار هيجانى \\
\hline$\cdot / \mu F$ & $.1 . .1$ & $19 / 941$ & GT/TA. & 1 & GT/KA. & سبك ارزيابى شناختى \\
\hline - ITFA &.$/ .1$ & $I T / V \Delta \cdot$ & $r q q / T \cdot q$ & 1 & $r q q / T \cdot q$ & تحمل بريشانى \\
\hline$\cdot 1 \cdot \vee 9$ & $>\cdot 1.91$ & $1 / 491$ & ITAT/KGD & 1 & IrAT/KGD & هايبندى مذهبى \\
\hline
\end{tabular}

فرا يند مقابله، مهارتهاى شناختى مورد استفاده قرار مى كيرد و افراد

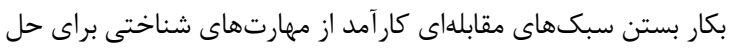

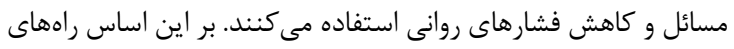

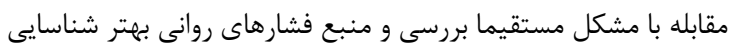

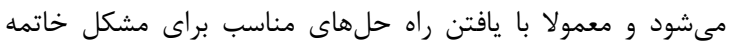

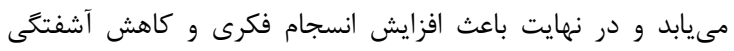

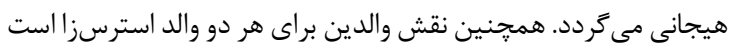

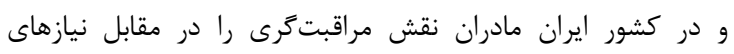

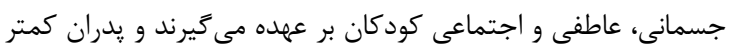

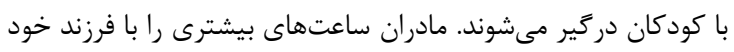

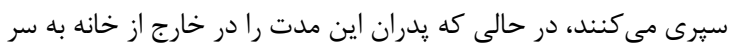

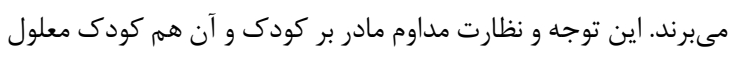

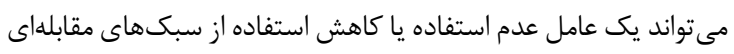

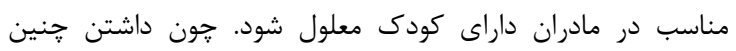

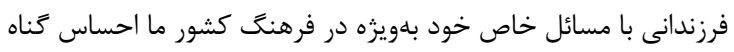

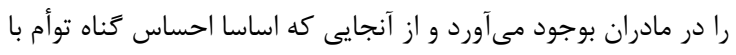

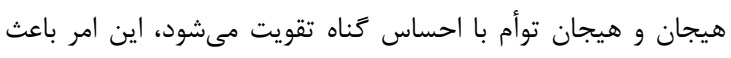

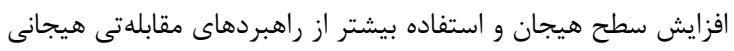

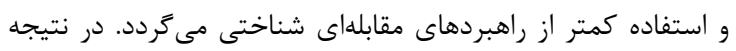

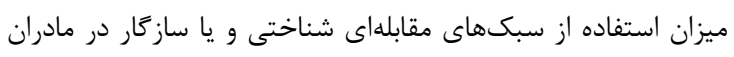

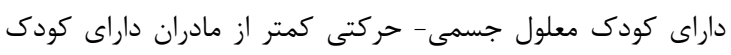
عادى است. ديكر نتايج نشان داد كه ميزان تحمل يريشانى مادران داراى كودى

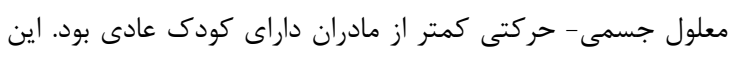

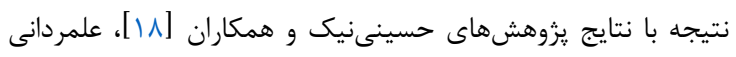

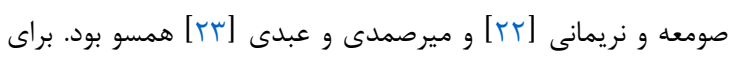

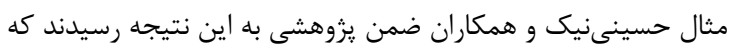

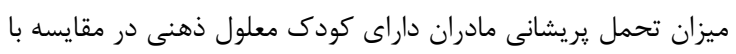

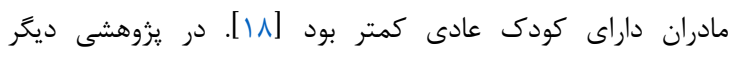

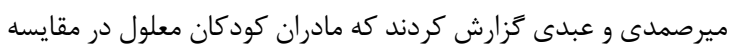

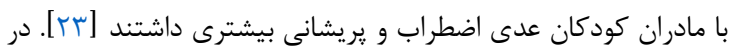

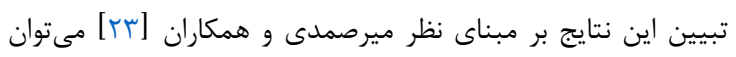

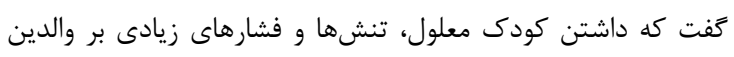

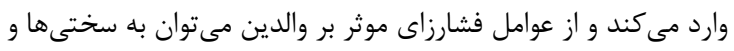

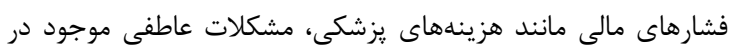

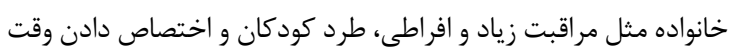

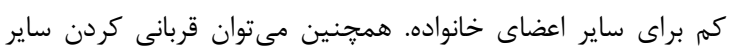

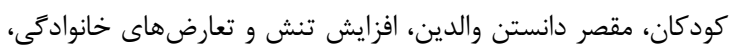

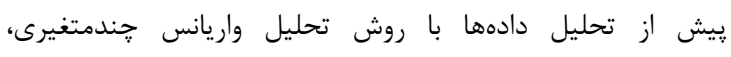

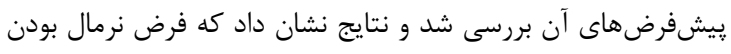

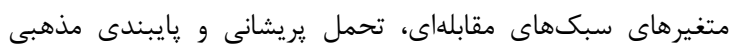

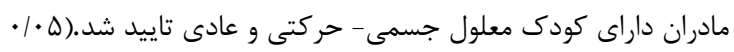

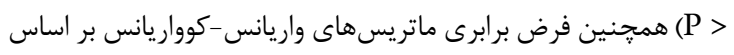

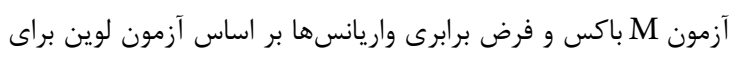

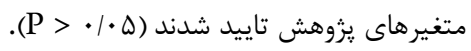

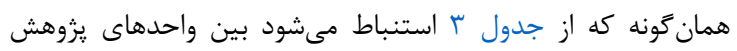

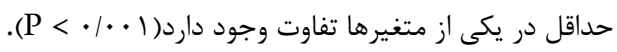

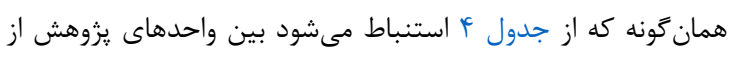

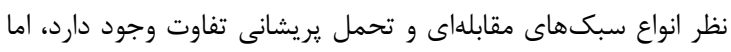

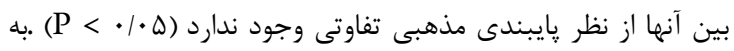

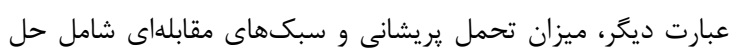

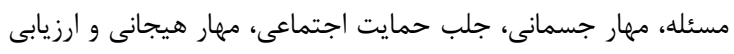

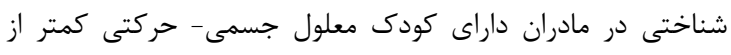

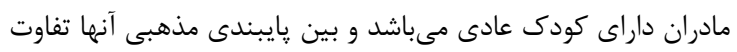

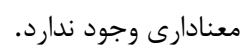

با توجه به آمار بالاى معلولان و بررسى تفاوتهاى والدين آنان با افراد

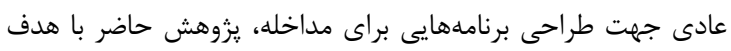

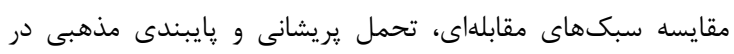

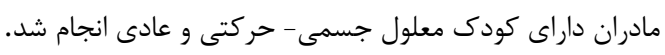

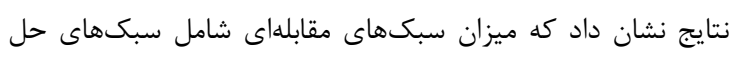

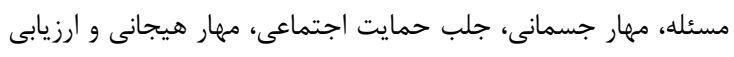

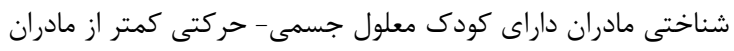

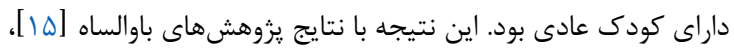

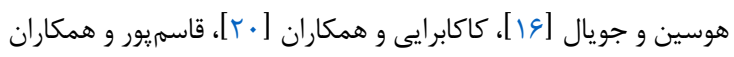

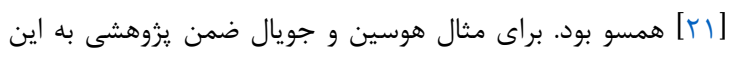
نتيجه رسيدند كه ميزان استفاده از سبكهاى مارئ مقابلهاى ساز

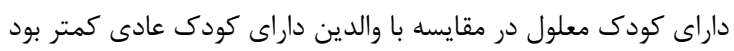

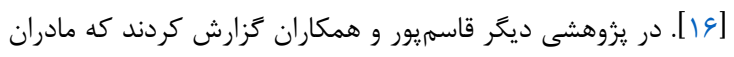
كودكان معلول شنوايى در مقايسه با مادران كودكان عادى بر براى مانى مقابله

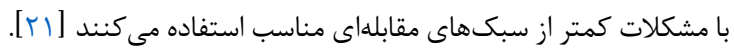

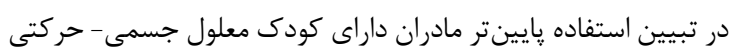

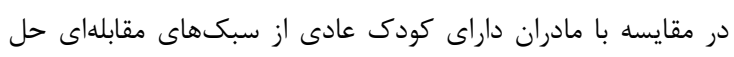

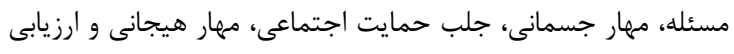

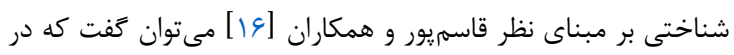


از محدوديتهاى يزوهش ميىتوان به محدود بودن آزمودنىها به شهر

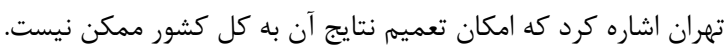

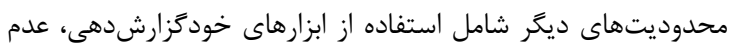

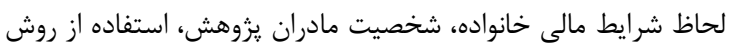

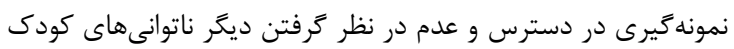

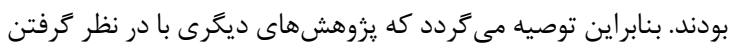

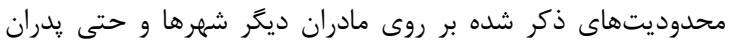

\section{نتيجه تيرى}

نتايج مطالعه حاضر نشان داد كه مادران داراى كودى معلول جسمى -

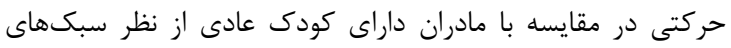

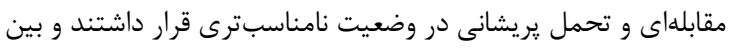

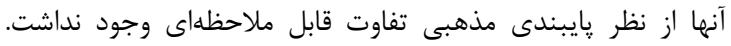

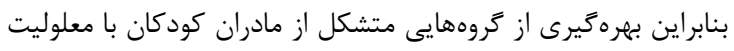

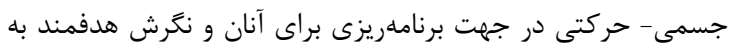

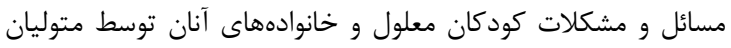
كودكان معلول در كشور نظير سازمان بهزيستى كشور مى تواند بر بهببود

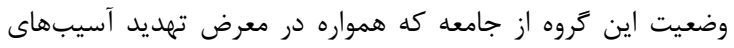

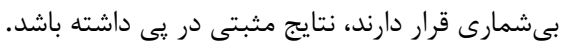

ملاحظات اخلاقى

در اين مطالعه، نكات اخلاقى طبق يروتكلهاتهاى اخلاقى مطالعات

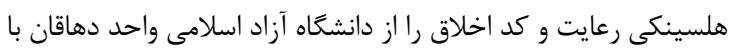

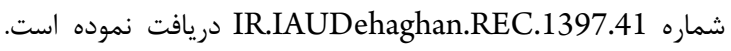
همجنين، فرم رضايتنامه شركت در يروهش توسط همه آزمونى هار نمده امضا شد.

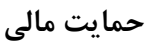
اين يزوهش حامى مالى نداشته و با هزينه شخصى انجام شده است. سهمم نويسندكًان جمع آورى دادهها، تحليل آنها و نغارش مقاله بر عهده زهرا ربانى بود. تضاد منافع در اين مقاله هيج تضاد منافعى گزارش نشده است. كاربرد عملى يافتهها

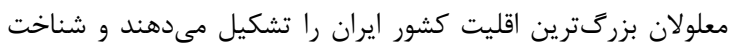

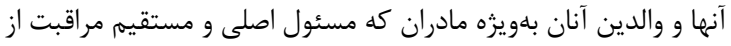

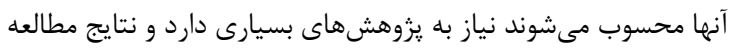

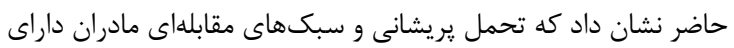

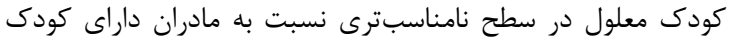

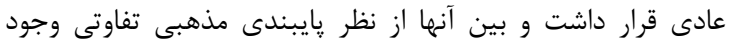

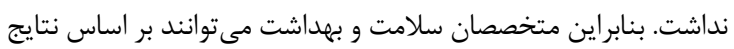

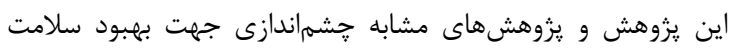

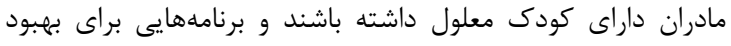

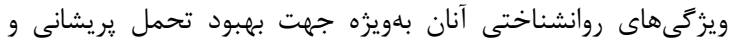

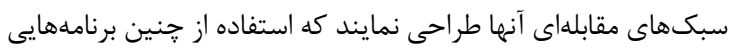

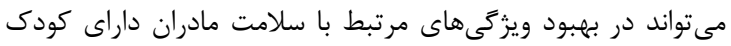

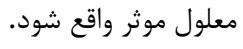

مشكلات و مسائل در روابط خانوادگى، عوامل فشارزاى ناشى از زندكى إنى

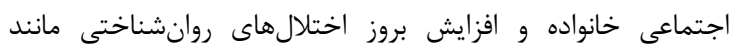

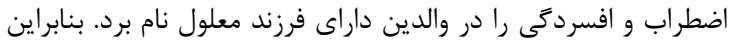

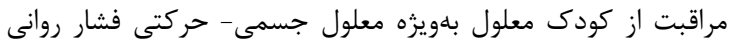

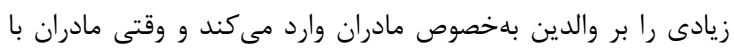

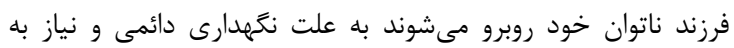

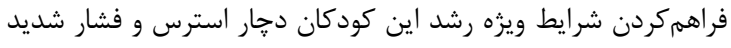

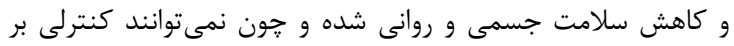
شرايط معلوليت كودى خود و فشارهاى واردشده بر زندگى خدى خود داشته

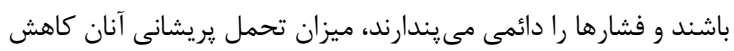
مى يابد. همجنين نتايج نشان داد كه ميزان گِايبندى مذهبى مادران داراى

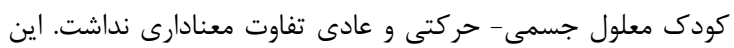

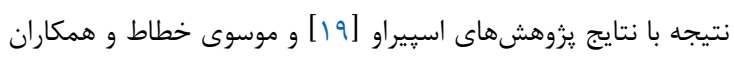

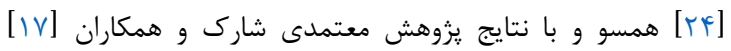

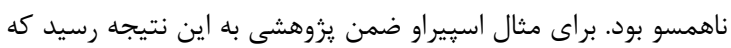

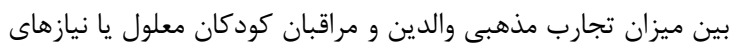

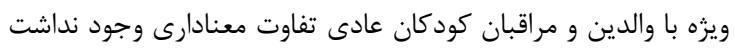

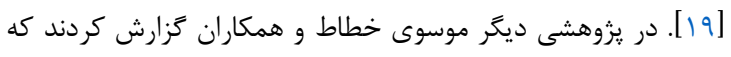

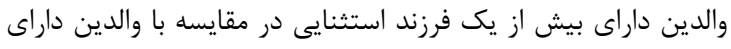

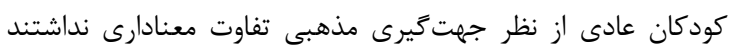

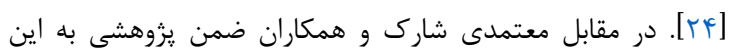

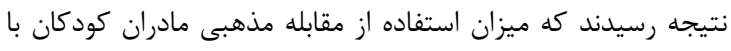

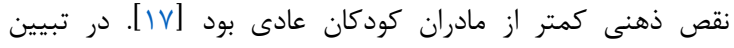

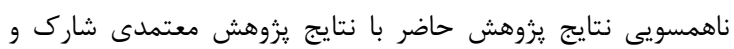

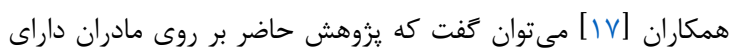

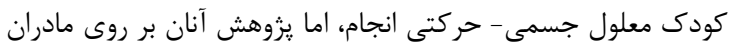

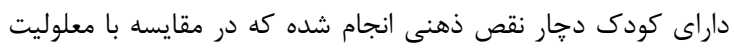

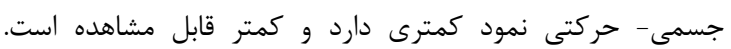

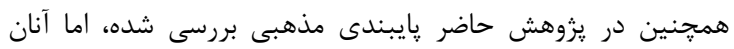

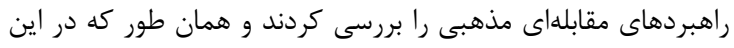

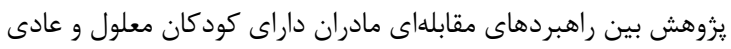

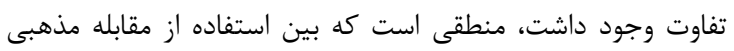

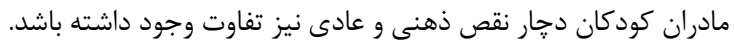

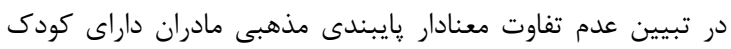

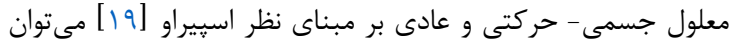

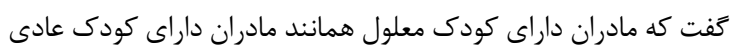

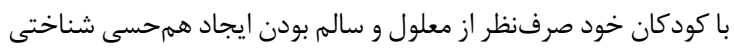

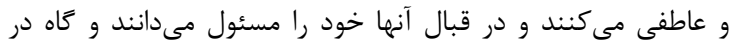

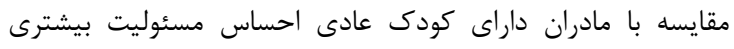

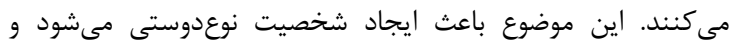

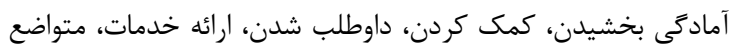

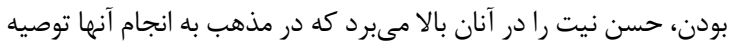

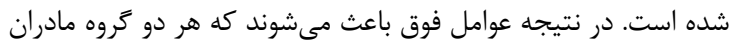

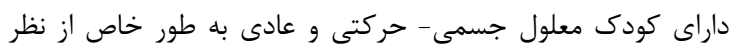

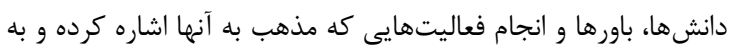
طور كلى از نظر پايبندى مذهبى تفاوت معنادارى نداشته باشند. 
بهزيستى شهر تهران به دليل همكارى جهت انجام يزوهش و از شركت كنند

\section{References}

1. Caron JG, Martin Ginis KA, Rocchi M, Sweet SN. Development of the Measure of Experiential Aspects of Participation for People With Physical Disabilities. Arch Phys Med Rehabil. 2019;100(1):67-77 e2. doi: 10.1016/j.apmr.2018.08.183 pmid: 30268805

2. De Hollander EL, Proper KI. Physical activity levels of adults with various physical disabilities. Prev Med Rep. 2018;10:370-6. doi: 10.1016/j.pmedr.2018.04.017 pmid: 29755934

3. Varsamis P, Agaliotis I. Relationships between gross- and fine motor functions, cognitive abilities, and selfregulatory aspects of students with physical disabilities. Res Dev Disabil. 2015;47:430-40. doi: 10.1016/j.ridd.2015.10.009 pmid: 26519661

4. Najmi B, Heidari Z, Feizi A, Hovsepian S, Momeni F, Azhar SMM. Do psychological characteristics of mothers predict parenting stress? A cross-sectional study among mothers of children with different disabilities. Arch Psychiatr Nurs. 2018;32(3):396-402. doi: 10.1016/j.apnu.2017.12.004 pmid: 29784221

5. $\mathrm{Qu}$ J, Fletcher QE, Reale D, Li W, Zhang Y. Independence between coping style and stress reactivity in plateau pika. Physiol Behav. 2018;197:1-8. doi: 10.1016/j.physbeh.2018.09.007 pmid: 30236525

6. Yang Y, Sun G, Dong X, Zhang H, Xing C, Liu Y. Preoperative anxiety in Chinese colorectal cancer patients: The role of social support, self-esteem and coping styles. J Psychosom Res. 2019;121:81-7. doi: 10.1016/j.jpsychores.2019.02.009 pmid: 30928212

7. Horwitz AG, Czyz EK, Berona J, King CA. Prospective associations of coping styles with depression and suicide risk among psychiatric emergency patients. Behav Ther. 2018;49(2):225-36. doi: 10.1016/j.beth.2017.07.010 pmid: 29530261

8. Veilleux JC. The relationship between distress tolerance and cigarette smoking: A systematic review and synthesis. Clin Psychol Rev. 2019;71:78-89. doi: 10.1016/j.cpr.2019.01.003 pmid: 30691959

9. Ellis AJ, Salgari G, Miklowitz D, Loo SK. Is distress tolerance an approach behavior? An examination of frontal alpha asymmetry and distress tolerance in adolescents. Psychiatry Res. 2018;267:210-4. doi: 10.1016/j.psychres.2018.05.083 pmid: 29933213

10. Peraza N, Smit T, Garey L, Manning K, Buckner JD, Zvolensky MJ. Distress tolerance and cessation-related cannabis processes: The role of cannabis use coping motives. Addict Behav. 2019;90:164-70. doi: 10.1016/j.addbeh.2018.10.047 pmid: 30408699

11. Matheny NL, Summers BJ, Macatee RJ, Harvey AM, Okey SA, Cougle JR. A multi-method analysis of distress tolerance in body dysmorphic disorder. Body Image. 2017;23:50-60. doi: 10.1016/j.bodyim.2017.07.005 pmid: 28826046
سبراسگذارى

12. Rupasingha A, Chilton JB. Religious adherence and county economic growth in the US. J Econ Behav Organ. 2009;72(1):438-50. doi: 10.1016/j.jebo.2009.05.020

13. Ahrenfeldt LJ, Moller S, Hvidt NC, Lindahl-Jacobsen R. Religiousness and lifestyle among Europeans in SHARE. Public Health. 2018;165:74-81. doi: 10.1016/j.puhe.2018.09.009 pmid: 30384031

14. Macilvaine WR, Nelson LA, Stewart JA, Stewart WC. Association of strength of religious adherence to quality of life measures. Complement Ther Clin Pract. 2013;19(4):251-5. doi: 10.1016/j.ctcp.2013.05.001 pmid: 24199983

15. Bawalsah JA. Stress and coping strategies in parents of children with physical, mental, and hearing disabilities in Jordan. Int J Educ. 2016;8(1):1-22. doi: 10.5296/ije.v8i1.8811

16. Hussain A, Juyal I. Stress appraisal and coping strategies among parents of physically challenged children. J India Acad Appl Psychol. 2007;33(2):179-82.

17. Motamedi Sharak F, Ghobari Bonab B, Jahed M. Relationship between stress and religious coping and mental health in mothers with normal and intellectually disabled children. Int J Educ Psychol Res. 2017;3(3):198204. doi: $10.4103 / 2395-2296.219422$

18. Hosseininik SS, Amiri F, MirzaeiRad R. Comparison of distress tolerance and adjustment of mothers of intellectual disability children and mothers of normal children in Yasouj city (Iran). NeuroQuantol. 2018;16(1):7-13. doi: 10.14704/nq.2018.16.1.1163

19. Speraw S. Spiritual experiences of parents and caregivers who have children with disabilities or special needs. Issues Ment Health Nurs. 2006;27(2):213-30. doi: 10.1080/01612840500436974 pmid: 16418080

20. Kakabaraee K, Afrooz GA, Hooman HA, Moradi A. Investigation and comparison of the mental wellbeing, coping styles and perceived social support in parents having exceptional and normal children. (Persian). RPH. 2012;5(2):45-58.

21. Ghasempour A, Akbari E, Taghipour M, Azimi Z, Refaghat E. Comparison of psychological well-being and coping styles in mothers of deaf and normally-hearing children. (Persian). Biomothly Audiol. 2012;21(4):51-9.

22. Almardani Some e, S., Narimani M. Comparison the intolerance of uncertainty and emotion regulation in mothers of deaf and hearing daughters. (Persian). Quarter Preschool Element School Stud. 2015; 1(1):120-40.

23. Mirsamadi F, Abdi R. Comparison of quality of life and mental health of mothers of children with disabilities and mothers of normal children. (Persian). MEJDS. 2017;7:17.

24. Mousavy-Khatat M, Soleimani M, A'bdi K. Comparison of cognitive, psychological and social characteristics, between parents who have more than one exceptional 
child with parents of normal child. (Persian). J Rehabi. 2011;12(3):53-64.

25. Satvat A, Khodabakhshi-Koolaee A, Falsafinejad MR, Sanagoo A. Title: Identifying the challenges marriage of person with physical-motor disabilities in Tehran: A phenomenological study. (Persian). IJRN. 2019;5(2):5562. doi: 10.21859/ijrn-05028

26. Atefvahid MK, Habibi Asgarabad M, Ashouri A, Sayed Alitabar SH. Parenting stress in mothers of mentally retarded, blind, deaf and physically disabled children. (Persian). Community Health. 2016;3(4):330-40.

27. Kadivar M, Kahrazei F, Nikmanesh Z. The effectiveness of interventions based on positive psychotherapy on improving coping styles, relapse prevention in individuals with substance use disorder. (Persian). Positive Psychol. 2016;1(4):41-54.

28. Simons JS, Gaher RM. The distress tolerance Scale: Development and validation of a self-report measure. Motivation Emotion. 2005;29(2):83-102. doi: 10.1007/s11031-005-7955-3

29. Safarzadeh S, Savari K, Dashtbozorgi Z. Comparison of distress tolerance, coping styles, spiritual intelligence and happiness among elderly men and women. (Persian). J Aging Psychol. 2017;2(4):237-48.

30. Janbozorgi M. Construction and validity of religious adherence test on the basis of the statements of Quran and Nahjul Balaghah. (Persian). Stud Islam Psychol. 2009;3(5):79-105 